\title{
THE EXPANSION THEOREM FOR PSEUDO-ANALYTIC FUNCTIONS ${ }^{1}$
}

\author{
SHMUEL AGMON AND LIPMAN BERS
}

1. Introduction. In this note we settle a question from the theory of pseudo-analytic functions which was left open in the original formulation of the theory $[2 ; 3] .^{2}$

We first recall the basic definitions. Let $F(z), G(z)$ be two complexvalued functions defined in a domain $\Omega$ of the $z=x+i y$ plane. $(F, G)$ is called a generating pair if the partial derivatives $F_{x}, G_{x}, F_{y}, G_{y}$ exist and satisfy a Hölder condition, and if $\operatorname{Im}(\bar{F} G)>0$. A function $w(z)$ defined in a subdomain $D \subset \Omega$ admits the unique representation

$$
w(z)=\phi(z) F(z)+\psi(z) G(z)
$$

with real $\phi$ and $\psi$. It is called regular pseudo-analytic in $D$ if at every point $z_{0}$ of $D$ the $(F, G)$-derivative

$$
\dot{w}\left(z_{0}\right)=\lim _{z \rightarrow z_{0}} \frac{w(z)-\phi\left(z_{0}\right) F(z)-\psi\left(z_{0}\right) G(z)}{z-z_{0}}
$$

exists (and is finite). The class of pseudo-analytic functions is closed under addition, multiplication by real constants, and bounded convergence.

The theory of pseudo-analytic functions parallels closely classical function theory to which it reduces for $F \equiv 1, G \equiv i$. It bears the same relationship to the general theory of linear partial differential equations of second order and elliptic type as classical function theory does to that of the Laplace equation. In fact, the function (1.1) is pseudo-analytic if and only if $\phi$ and $\psi$ satisfy the equation

$$
F\left(\phi_{x}+i \phi_{y}\right)+G\left(\psi_{x}+i \psi_{y}\right)=0
$$

and every linear equation

$$
a \Phi_{x x}+2 b \Phi_{x y}+c \Phi_{y y}+d \Phi_{x}+e \Phi_{y}+f \Phi=0, \quad a c-b^{2}>0,
$$

with sufficiently smooth coefficients $a(x, y), \cdots, f(x, y)$ can be re-

Presented to the Society, September 7, 1951; received by the editors February 11, 1952.

${ }^{1}$ The preparation of this paper was sponsored (in part) by the Office of Naval Research.

2 Numbers in brackets refer to the bibliography. We refer to [2] for the statement of results, to [3] for the proofs. A full presentation of this theory will appear elsewhere. 
duced, at least locally, to the form (1.2).

The generating pair $(F, G)$ is called normal if $\Omega$ is the whole finite $z$-plane, $F(z)$ and $G(z)$ are bounded, for some $\alpha>1$ the partial derivatives $F_{x}, \cdots, G_{y}$ are $O\left(|z|^{-\alpha}\right.$ ) as $z \rightarrow \infty$ (which implies that the limits $F(\infty), G(\infty)$ exist), and $\operatorname{Im}\{\bar{F}(\infty) G(\infty)\}>0 . w(z)$ is called pseudoanalytic at $z=\infty$ if it is single-valued, pseudo-analytic, and bounded for $R<|z|<+\infty$. This implies that the limit $w(\infty)$ exists.

In what follows we assume a fixed normal generating pair. Our aim is to establish the analogues of the Taylor and Laurent expansions for pseudo-analytic functions.

2. Similarity principle. Formal powers. A fundamental property of pseudo-analytic functions is expressed by the following theorem (stated here for the case of normal generating pairs).

SIMILARITY PRINCIPLE. Let $w(z)$ be single-valued and pseudo-analytic in a domain $D$, except perhaps for isolated points. Then there exists an analytic function which is regular in $D$, except perhaps for isolated points, and a function $S(z)$ which is continuous on the closure $\bar{D}$ of $D$, such that

$$
\begin{gathered}
w(z) \equiv S(z) f(z), \\
0<1 / K \leqq|S(z)| \leqq K
\end{gathered}
$$

where $K$ is a constant depending only on $(F, G)$. Conversely, if $f(z)$ is single-valued and regular analytic in $D$, except perhaps at isolated points, then there exists a function $S(z)$ satisfying the preceding conditions such that $w(z)$ defined by (2.1) is pseudo-analytic at all regular points of $f(z)$ in $D$. If one of the functions $f$ or $w$ is given, $S$ may be chosen so that $S\left(z_{0}\right)=1$ at a preassigned point $z_{0}$ of $\bar{D}$.

Applying this theorem to the function $f(z)=a\left(z-z_{0}\right)^{n}$, where $n$ is an integer and $a \neq 0$, and taking for $D$ the finite $z$-plane, we conclude that there exists a pseudo-analytic function

$$
w(z)=Z^{(n)}\left(a, z_{0} ; z\right)
$$

such that

$$
\begin{aligned}
& Z^{(n)}\left(a, z_{0} ; z\right) \sim a\left(z-z_{0}\right)^{n}, \quad z \rightarrow z_{0}, \\
& \frac{1}{K} \leqq\left|\frac{Z^{(n)}\left(a, z_{0} ; z\right)}{a\left(z-z_{0}\right)^{n}}\right| \leqq K .
\end{aligned}
$$

It is easy to show that the function (2.3) is determined uniquely. We also set 


$$
Z^{(n)}\left(0, z_{0} ; z\right) \equiv 0 \text {. }
$$

The global formal powers defined above satisfy the relations

$$
Z^{(0)}\left(a, z_{0} ; z\right)=\lambda F(z)+\mu G(z), \quad \lambda F\left(z_{0}\right)+\mu G\left(z_{0}\right)=a
$$

where $\lambda$ and $\mu$ are real constants, and

$$
Z^{(n)}\left(a^{\prime}+i a^{\prime \prime}, z_{0} ; z\right)=a^{\prime} Z^{(n)}\left(1, z_{0} ; z\right)+a^{\prime \prime} Z^{(n)}\left(i, z_{0} ; z\right),
$$

if $a^{\prime}$ and $a^{\prime \prime}$ are real.

The similarity principle also implies the existence of pseudoanalytic functions

$$
w(z)=Z^{(n)}\left(a, z_{0} ; z\right)
$$

("global formal power normalized at infinity") satisfying the relations

$$
\tilde{Z}^{(n)}\left(0, z_{0} ; z\right) \equiv 0
$$

and for $a \neq 0$

$$
\begin{gathered}
Z^{(n)}\left(a, z_{0} ; z\right) \sim a z^{n}, \quad z \rightarrow \infty, \\
\frac{1}{K} \leqq\left|\frac{Z^{(n)}\left(a, z_{0} ; z\right)}{a\left(z-z_{0}\right)^{n}}\right| \leqq K .
\end{gathered}
$$

It is not difficult to show that

$$
\tilde{Z}^{(n)}\left(a, z_{0} ; z\right)=Z^{(n)}\left(\tilde{a}, z_{0} ; z\right)
$$

where $\tilde{a}$ depends on $a, z_{0}$, and $n$, and $\tilde{a}=0$ if and only if $a=0$.

3. The expansion theorem. Taylor coefficients. We shall prove the following:

EXPANSION THEOREM. Let $w(z)$ be a regular pseudo-analytic function defined for $\left|z-z_{0}\right|<R$. Then it admits a unique expansion of the form

$$
w(z)=\sum_{n=0}^{\infty} Z^{(n)}\left(\alpha_{n}, z_{0} ; z\right)
$$

which converges for $\left|z-z_{0}\right|<R$.

Consider first an arbitrary series

$$
\sum_{n=0}^{\infty} Z^{(n)}\left(\alpha_{n}, z_{0} ; z\right)
$$

It follows from (2.5) that this series converges uniformly and ab- 
solutely in every closed subdomain within the circle of convergence of the series

$$
\sum_{n=0}^{\infty} \alpha_{n}\left(z-z_{0}\right)^{n}
$$

(provided it has a positive radius), and diverges at every point outside this circle (provided the series (3.3) does not converge everywhere). Assume now that (3.2) converges for some $z \neq z_{0}$. Its sum, say $w(z)$, is then pseudo-analytic in some neighborhood of $z_{0}$ and it follows from (2.4) that

$$
\begin{gathered}
\alpha_{0}=w\left(z_{0}\right), \\
\alpha_{n}=\lim _{z \rightarrow z_{0}} \frac{w(z)-\sum_{\nu=1}^{n-1} Z^{(\nu)}\left(\alpha_{\nu}, z_{0} ; z\right)}{\left(z-z_{0}\right)^{n}}, n=1,2, \cdots .
\end{gathered}
$$

This implies the uniqueness part of the expansion theorem.

Next, let $w(z)$ be any pseudo-analytic function defined in the neighborhood of $z_{0}$. By virtue of the similarity principle, if $w\left(z_{0}\right)=0$ and $w(z) \neq 0$, then $w(z) \sim a\left(z-z_{0}\right)^{n}$ for some $a \neq 0$ and some positive integer $n$. We conclude that the numbers $\left\{\alpha_{n}\right\}$ given by (3.4) exist. We call them the Taylor coefficients of $w(z)$ at $z_{0}$. It is clear that the Taylor coefficients depend linearly on the function, that is, that if $w^{*}(z)$ has the Taylor coefficients $\left\{\alpha_{n}^{*}\right\}$, the Taylor coefficients of $\lambda w(z)+\mu w^{*}(z)$ are $\left\{\lambda \alpha_{n}+\mu \alpha_{n}^{*}\right\}, \lambda$ and $\mu$ being real constants. Also, $\alpha_{0}=\alpha_{1}=\cdots=0$ if and only if $w(z) \equiv 0$. Hence if the coefficients $\alpha_{n}$ of (3.2) are the Taylor coefficients of $w(z),(3.1)$ holds within the circle of convergence of (3.3).

The following statement is proved in [2].

Lemma A. Let w(z) be pseudo-analytic and bounded by $M$ in absolute value for $\left|z-z_{0}\right|<R$. Let $\left\{\alpha_{n}\right\}$ be defined by (3.4). Then

$$
\left|\alpha_{n}\right| \leqq M\left(\frac{K_{1}}{R}\right)^{n}
$$

where $K_{1}$ is a positive constant depending only on $(F, G)$.

In view of what was said above, this implies a weaker form of the expansion theorem, with the convergence of (3.1) established only for $\left|z-z_{0}\right|<R / K_{1}$. On the other hand, the expansion theorem will be completely proved once we verify the following lemma.

Lemma B. Under the hypotheses of Lemma A 


$$
\left|\alpha_{n}\right| \leqq \frac{M K}{R^{n}}
$$

where $K$ is the constant occurring in the statement of the similarity principle.

4. Proof of the expansion theorem. We first establish the following lemma.

Lemma C. For some fixed $a, z_{0}, \zeta \neq z_{0}$ let $\left\{\beta_{n}\right\}$ be the Taylor coefficients of the function $Z^{(-1)}(a, \zeta ; z)$ at $z_{0}$. Then

$$
\frac{|a|}{K\left|\zeta-z_{0}\right|^{n+1}} \leqq\left|\beta_{n}\right| \leqq \frac{|a| K}{\left|\zeta-z_{0}\right|^{n+1}} .
$$

We assume that $a \neq 0$ (otherwise there is nothing to prove) and for some fixed $n>0$ consider the pseudo-analytic function

$$
W(z)=Z^{(-1)}(a, \zeta ; z)-\sum_{\nu=0}^{n-1} Z^{(\nu)}\left(\beta_{\nu}, z_{0} ; z\right) .
$$

This function is regular everywhere except at $z=\zeta$ and $z=\infty$ where

$$
\begin{aligned}
W(z) & \sim \frac{a}{z-\zeta}, & & z \rightarrow \zeta, \\
W(z) & =O\left(|z|^{n-1}\right), & & \rightarrow \infty .
\end{aligned}
$$

By virtue of the similarity principle we may state that $W(z)=S(z) q(z)$ where

$$
S(\zeta)=1, \quad 1 / K \leqq|S(z)| \leqq K,
$$

and $q(z)$ is a rational function of degree at most $n$. Since

$$
W(z)=O\left(\left|z-z_{0}\right|^{n}\right), \quad z \rightarrow z_{0},
$$

we have that

$$
q(z)=\frac{a\left(z-z_{0}\right)^{n}}{(z-\zeta)\left(\zeta-z_{0}\right)^{n}}
$$

But

$$
\beta_{n}=\lim _{z \rightarrow z_{0}} \frac{W(z)}{\left(z-z_{0}\right)^{n}}=-\frac{a S\left(z_{0}\right)}{\left(\zeta-z_{0}\right)^{n+1}}
$$

which implies (4.1). For $n=0$ the statement follows directly from (2.5). 
In order to prove Lemma B we may assume without loss of generality that $w(z)$ is continuous for $\left|z-z_{0}\right| \leqq R$. Now if a pseudo-analytic function is continuous on the closure of a bounded domain $D$ bounded by a piecewise continuously differentiable curve $\Gamma$, then the Cauchy formula

$$
w(z)=-\int_{\Gamma} Z^{(-1)}\left\{\frac{w(\zeta) d \zeta}{2 \pi i}, \zeta ; z\right\}
$$

holds for $z \in D$. The integral on the right-hand side is to be interpreted as

$$
-\int_{0}^{L} Z^{(-1)}\left\{\frac{w[\zeta(s)] \zeta^{\prime}(s)}{2 \pi i}, \zeta(s) ; z\right\} d s
$$

where

$$
\zeta=\zeta(s), \quad 0 \leqq s \leqq L,
$$

is the parametric representation of $\Gamma$ in terms of its arc-length. Applying this to the domain $\left|z-z_{0}\right| \leqq R$, and representing the integral as a limit of Riemann sums, we see that

$$
w(z)=\lim _{N \rightarrow \infty} w_{N}(z)
$$

where

$$
w_{N}(z)=\frac{1}{N} \sum_{\nu=1}^{N} Z^{(-1)}\left\{w\left(z_{0}+R e^{2 \pi i \nu / N}\right) R e^{2 \pi i \nu / N}, z_{0}+R e^{2 \pi i \nu / N} ; z\right\} .
$$
C

Let $\left\{\alpha_{n}^{(N)}\right\}$ be the Taylor coefficients of $w_{N}(z)$ at $z=z_{0}$. By Lemma

$$
\left|\alpha_{n}^{(N)}\right| \leqq \frac{K M}{R^{n}}
$$

But by (2.5) we have that $\left|w_{N}(z)\right| \leqq 2 K M$ for $\left|z-z_{0}\right| \leqq R / 2$, and since bounded convergence of pseudo-analytic functions implies uniform convergence in every closed subdomain, (4.2) holds uniformly for $\left|z-z_{0}\right|<R / 3$. Applying Lemma A to the difference $\left[w(z)-w_{N}(z)\right]$ and the domain $\left|z-z_{0}\right|<R / 3$, we conclude that

$$
\lim _{N \rightarrow \infty} \alpha_{n}^{(N)}=\alpha_{n}
$$

so that Lemma B (and hence also the expansion theorem) follows from (4.3). 
5. Laurent series. Let $w(z)$ be pseudo-analytic for $\left|z-z_{0}\right|>R$ (that is, also at $z=\infty$ ). Using the method of $\$ \S 3,4$ we can show that

$$
w(z)=\sum_{n=0}^{\infty} \tilde{Z}^{(-n)}\left(\gamma_{n}, z_{0} ; z\right),
$$

the series converging for $\left|z-z_{0}\right|>R$, and the coefficients being given by

$$
\begin{gathered}
\gamma_{0}=w(\infty), \\
\gamma_{n}=\lim _{z \rightarrow \infty}\left\{z^{n}\left[w(z)-\sum_{\nu=0}^{n-1} \tilde{Z}^{(\nu)}\left(\gamma_{\nu}, z_{0} ; z\right)\right]\right\}, \quad n=1,2, \cdots .
\end{gathered}
$$

In view of (2.6) the expansion (5.1) may also be written in the form

$$
w(z)=\sum_{n=0}^{\infty} Z^{(-n)}\left(\alpha_{n}, z_{0} ; z\right) .
$$

Now let $w(z)$ be single-valued and pseudo-analytic for $R_{1}<\left|z-z_{0}\right|$ $<R_{2}$. It is known (and easy to show using the Cauchy formula referred to in the preceding section) that $w(z)$ admits the unique representation $w(z)=w_{1}(z)+w_{2}(z), w_{1}(z)$ and $w_{2}(z)$ being regular pseudoanalytic functions defined for $\left|z-z_{0}\right|>R_{1}$ and $\left|z-z_{0}\right|<R_{2}$, respectively, and such that $w_{1}(\infty)=0$. Using the preceding results we obtain the following theorem.

EXPANSION THEOREM FOR THE RING. Let $w(z)$ be a single-valued pseudo-analytic function defined for $R_{1}<\left|z-z_{0}\right|<R_{2}$. Then it admits a unique expansion of the form

$$
w(z)=\sum_{n=-\infty}^{+\infty} Z^{(n)}\left(\alpha_{n}, z_{0} ; z\right),
$$

converging for $R_{1}<\left|z-z_{0}\right|<R_{\mathbf{2}}$.

The expansion near an isolated singularity $\left(R_{1}=0\right.$ or $\left.R_{2}=+\infty\right)$ is included in this result.

6. Remarks. (1) The Taylor coefficients of a pseudo-analytic function can be defined by formulas involving higher $(F, G)$-derivatives, instead of the formulas (3.4). We prefer here the present definition since it extends to the case $z_{0}=\infty$, and also to the case when at $z_{0}$ the generators $(F, G)$ fail to have derivatives but behave so that the similarity principle still holds.

(2) We emphasize that in this note we deal with global formal powers only. Expansion theorems involving local formal powers (for 
instance the theorems of [5] and [4]) are independent of the present results.

(3) Our original proof of the expansion theorem was based on the method of "principal coefficient sequences" [1]. While we could dispense with this method here, it will be of importance in obtaining deeper results (like gap theorems) for series of the form (3.1). These questions will be considered separately.

\section{REFERENCES}

1. S. Agmon, Sur les séries de Dirichlet, Ann. Ecole Norm. vol. 66 (1949) pp. 263310.

2. L. Bers, Partial differential equations and generalized analytic functions, Proc. Nat. Acad. Sci. U.S.A. vol. 36 (1950) pp. 130-136. Second note, ibid. vol. 37 (1951) pp. 42-47.

3. - Theory of pseudo-analytic functions (Mimeographed notes), New York University. To appear.

4. - The expansion theorem for sigma-monogenic functions. Amer. J. Math. vol. 72 (1950) pp. 705-711.

5. L. Bers and A. Gelbart, On a class of functions defined by partial differential equations, Trans. Amer. Math. Soc. vol. 56 (1943) pp. 168-188.

RICE INSTITUTE,

NeW YoRK UNIVERSITY, AND

National Bureau of Standards, Los Angeles 